\title{
Microstructure Observation of High-Current Buried-Arc Welded Joint ${ }^{*}$
}

\author{
by BABA Hayato **, HONDA Reo ***, ERA Tetsuo **, KOMEN Hisaya ****, \\ TANAKA Manabu $* * * * *$ and TERASAKI Hidenori $* * * * * *$
}

\begin{abstract}
To address the recent need for improved efficiency in thick plate welding, a high-current buried-arc welding system has been developed. In a high-current buried-arc welding process, it is necessary to adequately evaluate the thermal effect of high heat input on the welded joint. The mechanical properties have been verified ${ }^{1)}$, and in the present study, we focused on observing microstructures. In some regions of the weld metal in the joint welded through high-current buried-arc welding, a characteristic metallographic structure was observed in which the macrostructure appeared extremely coarse. However, this coarse macrostructure was assumed to be a trace of the prior $\gamma$ grain; the actual microstructure mainly consisted of fine intragranular idiomorphic ferrite and acicular ferrite. These two types of ferrite are known to precipitate at different temperature ranges; thus, their mixed structure is extremely characteristic and is formed because of high heat input.
\end{abstract}

Key Words: Buried Arc, Gas Metal Arc Welding, High Heat Input, Low-Carbon Steel, Thick Plate, Weld Metal, Crystal Orientation, Microstructure, Acicular Ferrite, Intragranular Idiomorphic Ferrite

\section{Introduction}

To meet the recent demand for thick plate welding with enhanced efficiency, a part of authors have developed a high-efficiency thick-plate welding system, which uses a high-current buried arc stabilized by the welding current and arc voltage waveforms control ${ }^{2}$. With this system, it became possible to achieve single-pass full penetration welding with a plate thickness of $19 \mathrm{~mm}$ at $60 \mathrm{~kJ} / \mathrm{cm}$ or higher. However, it is necessary to adequately evaluate the thermal effect of the high heat input on the welded joint.

By examining the metallographic structure of the welded joint, it was found that a characteristic metallographic structure appears, depending on the material and welding conditions. In this paper, we show the crystallographic feature of the weld metal formed by using high-current buried arc and discuss the formation process of the abovementioned metallographic structure.

\section{Experimental procedure}

\subsection{Production of Welded Joint}

By performing high-current buried-arc welding, a single-pass full penetration welded joint was prepared with a plate thickness

* Received: 2019.11.21, Presented at Visual-JW 2019

** Member, DAIHEN Corporation

*** Graduate School of Engineering, Kumamoto University

**** Member, Magnesium Research Center, Kumamoto University

***** Member, Joining and Welding Research Institute, Osaka University

${ }^{* * * * * *}$ Member, Graduate School of Engineering, Kumamoto University of $19 \mathrm{~mm}$. Table 1 lists the materials used and the welding conditions. Fig. 1 shows a schematic image of the welding system and Fig. 2 shows a schematic diagram of the welded joint including the groove shape. The high-current buried-arc was stabilized by low-frequency modulated voltage control ${ }^{2}$, and the welding current and arc voltage waveforms at the time of welding are shown in Fig. 3.

Table 1 Materials and Welding Conditions

\begin{tabular}{l||l}
\hline Base metal & JIS G 3106: 2015, SM490A \\
\hline Welding wire & JIS Z 3312: 2009, YGW11, $\varphi 1.4$ \\
\hline Welding current & $580 \mathrm{~A}$ \\
\hline Arc voltage & $44.5 \mathrm{~V}$ \\
\hline Wire feeding speed & $20.2 \mathrm{~m} / \mathrm{min}$ \\
\hline Welding speed & $24 \mathrm{~cm} / \mathrm{min}$ \\
\hline Heat input & $64.5 \mathrm{~kJ} / \mathrm{cm}$ \\
\hline Shielding gas & $\mathrm{CO}_{2}, 30 \mathrm{~L} / \mathrm{min}$ \\
\hline Contact tip-base metal & $20 \mathrm{~mm}$ \\
surface distance & \\
\hline
\end{tabular}

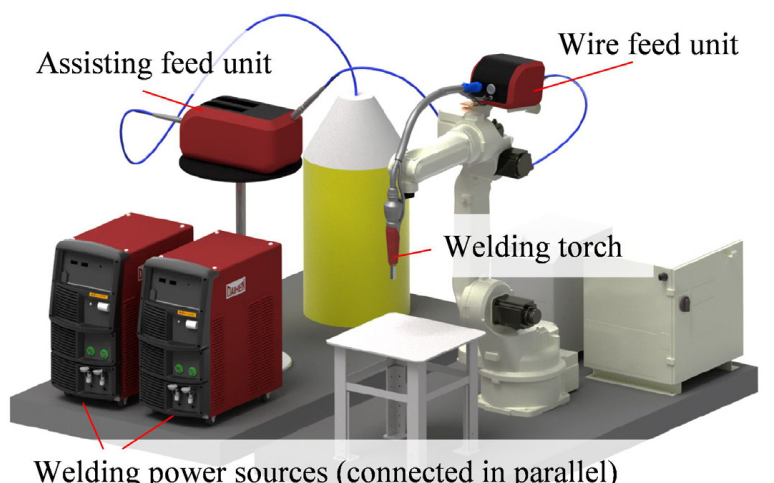

Welding power sources (connected in parallel)

Fig. 1 Schematic Image of Welding System 


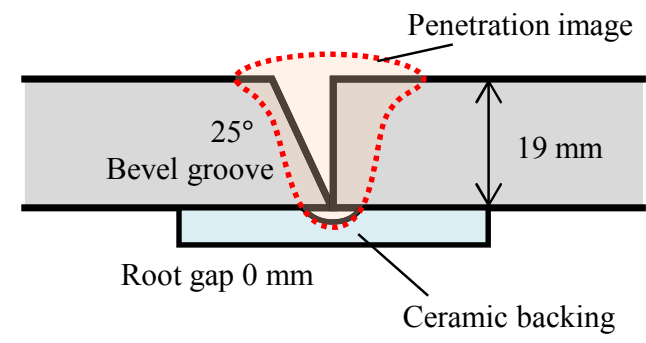

Fig. 2 Schematic Diagram of Welded Joint

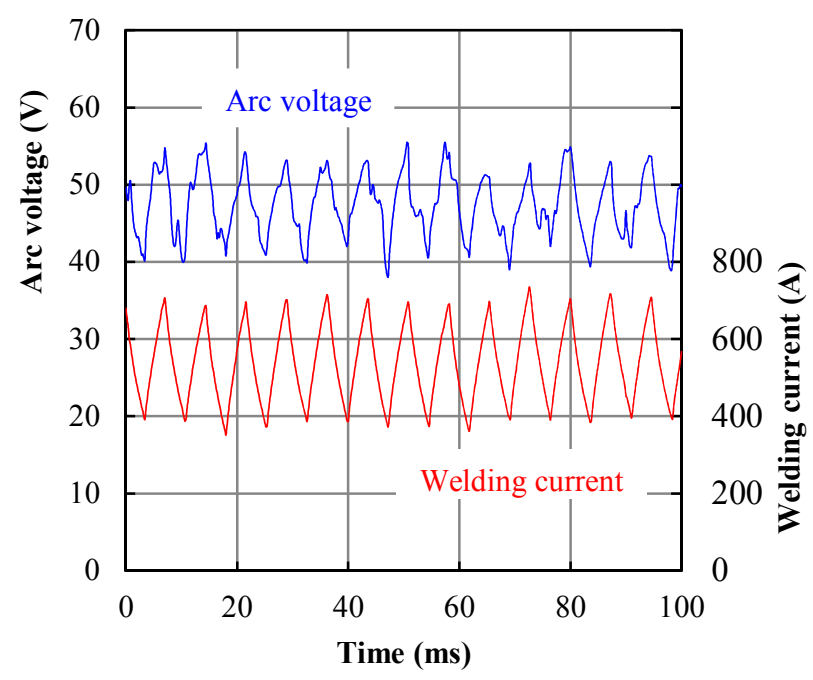

Fig. 3 Welding Current and Arc Voltage Waveforms

\subsection{Optical Microscopy Observation}

An optical microscope was used to observe the welded joint. Observation was carried out after polishing the specimen and etching with $3 \%$ nital.

\subsection{Analysis by Electron Backscatter Diffraction}

Electron backscatter diffraction (EBSD) was used to observe the microstructure and perform crystal orientation analysis of the welded joint. The electron acceleration voltage was $15 \mathrm{kV}$, and the step size was $0.2 \mu \mathrm{m}$.

\section{Results and Discussions}

Figure 4 shows the observation result for the macrostructure in the cross section of the welded joint. The weld metal was divided into three regions with different macrostructures, labeled (A), (B), and $(\mathrm{C})$ from the fusion boundary side to the weld metal center.

Fig. 5 shows the microstructures of the weld metal observed with an optical microscope. To observe the microstructure of each region in further detail, we used EBSD to construct $\alpha$-orientation maps and grain maps. The $\alpha$-orientation maps are shown in Fig. 6, and the grain maps on the same scale are shown in Fig. 7.

As shown in Fig. 4, the macrostructure in region (A) appears extremely coarse; however, microstructure observations reveal fine metallographic structures (Fig. 5 and Fig. 7). Fig. 6 shows a wider region in which coarse and fine ferrites are observed, where the latter are dominant. Coarse ferrites are likely grain-boundary ferrite that precipitated in the prior $\gamma$ grain boundary, i.e., grain-boundary allotriomorphic ferrite and secondary Widmanstätten ferrite ${ }^{3)}$. Because these ferrites are distributed along the outline of the macrostructure with a coarse appearance, this coarse appearance is assumed to be the trace of prior $\gamma$ grain. Meanwhile, the fine ferrites are likely intragranular ferrites with acicular ferrite inclusions, which precipitate within prior $\gamma$ grains.

In region (B), the microstructure components are the same as those in region (A): coarse grain-boundary ferrite and fine intragranular ferrite. However, there is less intragranular ferrite in region (B) than in region (A), and the ratio of grain-boundary ferrite is high because the prior $\gamma$ grains in region (B) are smaller than those in region (A), which leads to more prior $\gamma$ grain boundaries.

Region (C), i.e., the weld metal center, contains even less intragranular ferrite and grain-boundary ferrite than region (B); instead, extensive round and equiaxed ferrite textures are observed.

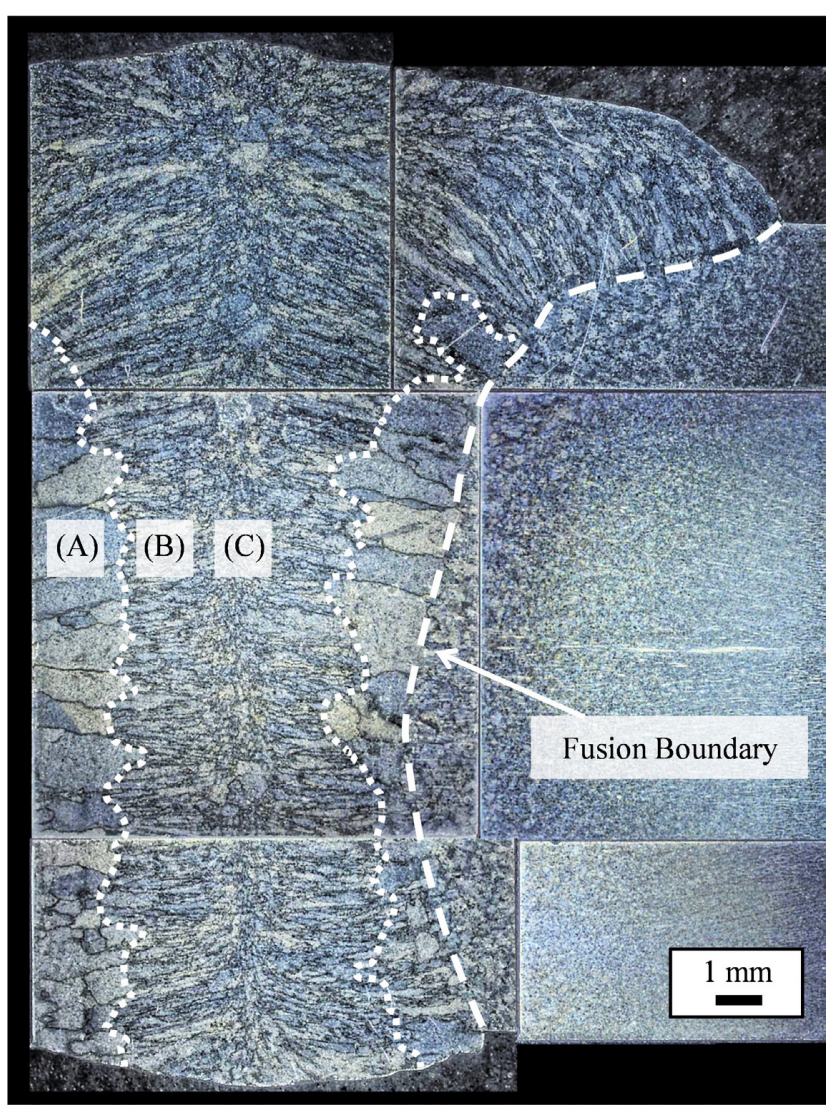

Fig. 4 Cross-Sectional Macrostructure of Welded Joint 


\begin{tabular}{|c|c|c|c|}
\hline & Region (A) & Region (B) & Region (C) \\
\hline & 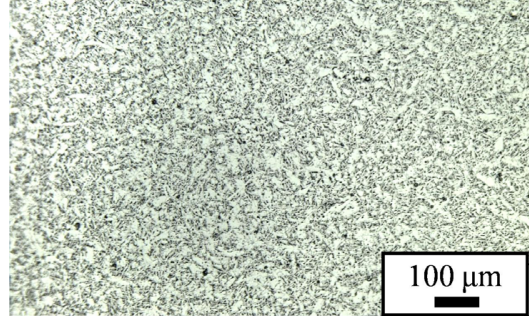 & 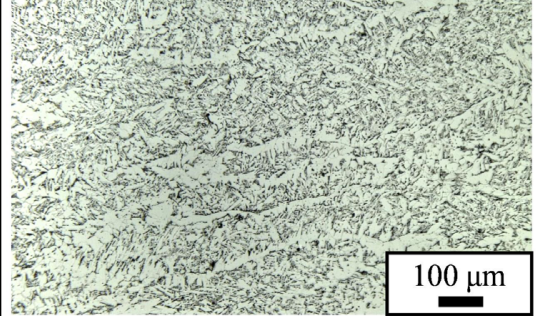 & 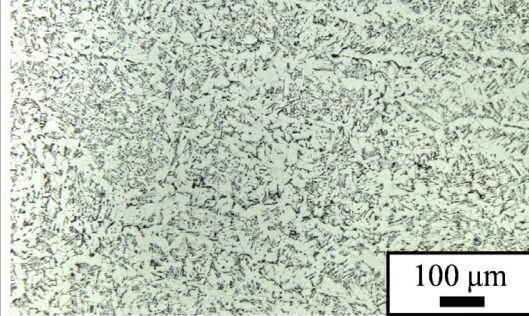 \\
\hline 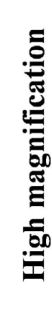 & 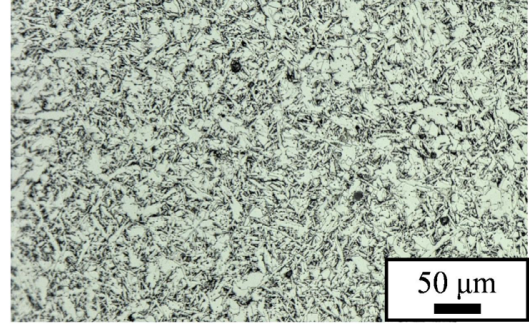 & 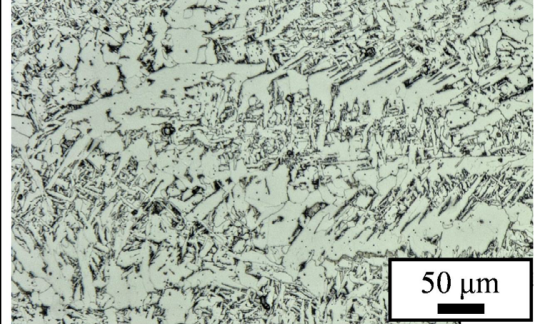 & 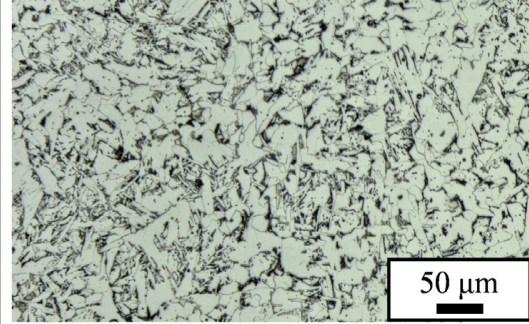 \\
\hline
\end{tabular}

Fig. 5 Microstructures of Weld Metal Observed with Optical Microscope

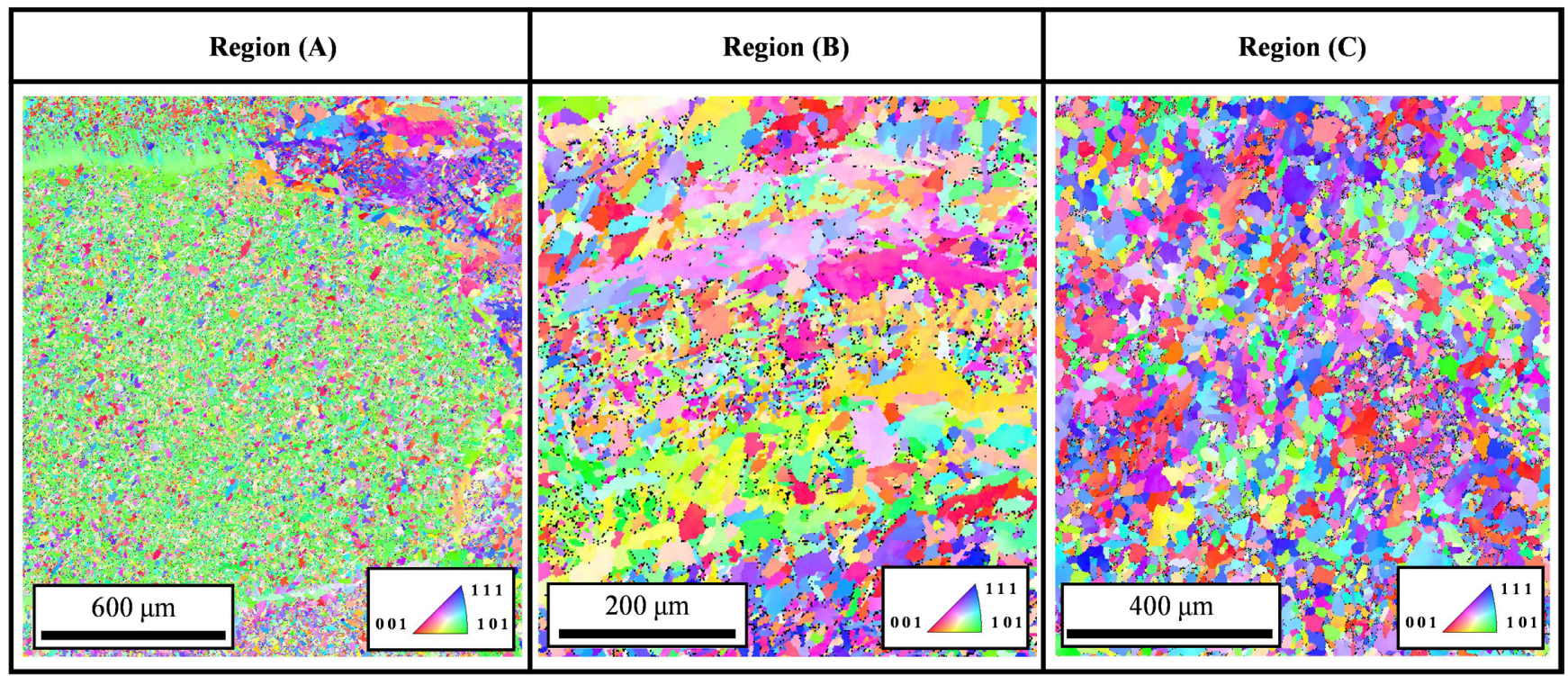

Fig. $6 \alpha$-Orientation Maps of Ferrite in Weld Metal

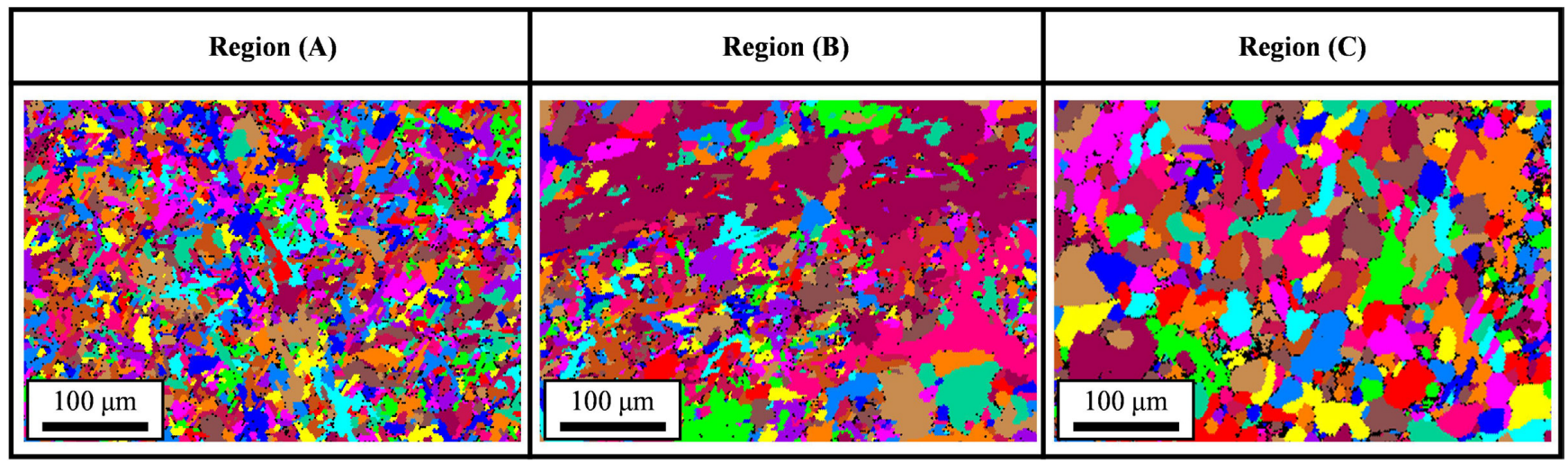

Fig. 7 Grain Maps of Ferrite in Weld Metal 
For a more detailed observation of region (A), which contains a particularly characteristic metallographic structure as a weld metal, we analyzed the variant group in the Kurdjumov-Sachs crystal orientation relationship (K-S OR) of the precipitated ferrite by EBSD. Fig. 8 (a) shows the image quality (IQ) map of a part of the weld metal and the region where the crystal orientation analysis was performed. The pole figure of $<001>\alpha$ is shown in Fig. 8 (b). A typical pattern that shows the K-S OR between $\alpha$ and $\gamma^{4)}$ can be observed, indicating that these ferrites have the $\mathrm{K}-\mathrm{S}$ OR with the prior $\gamma$ grain, and the crystal orientation of the prior $\gamma$ grain can be easily presumed. However, Fig. 8 (b) shows many plots that do not have the K-S OR. This pole figure was used for constructing a Bain map showing the variant group known as Bain group ${ }^{5,6}$. The Bain map is shown in Fig. 8 (c). The white parts indicate ferrites that do not satisfy the K-S OR with the prior $\gamma$ grain. These parts are considered intragranular idiomorphic ferrites and are known to precipitate at relatively high temperatures ${ }^{7}$. On the basis of the morphology and satisfying the K-S OR with the prior $\gamma$ grain, the other crystal grains colored in Fig. 8 (c) are believed to consist of acicular ferrite. Acicular ferrite is known to precipitate at relatively low temperatures ${ }^{7}$. There are few research reports on the mixed structure of these two phases whose precipitation temperatures differ significantly. For example, a research report on a metallographic structure of a relatively low heat input welded joint with a total heat input of $4.94 \mathrm{~kJ} / \mathrm{cm}$ using a laser and buried arc hybrid process shows that grain-boundary ferrite and acicular ferrite were observed, however, intragranular idiomorphic ferrite which precipitates at high temperatures was not observed ${ }^{8)}$. Therefore, the mixed structure of intragranular idiomorphic ferrite and acicular ferrite in region (A) is considered to be a characteristic microstructure in a high-current buried-arc welded joint.

Because the solidification in region (A) was relatively early and the high-temperature arc was still in the vicinity during the solidification, it is assumed that region (A) was maintained at high temperature for a long duration. Therefore, it is believed that the prior $\gamma$ grains became coarse, and intragranular idiomorphic



(a) IQ Map and Analyzed Prior $\gamma$ Grain

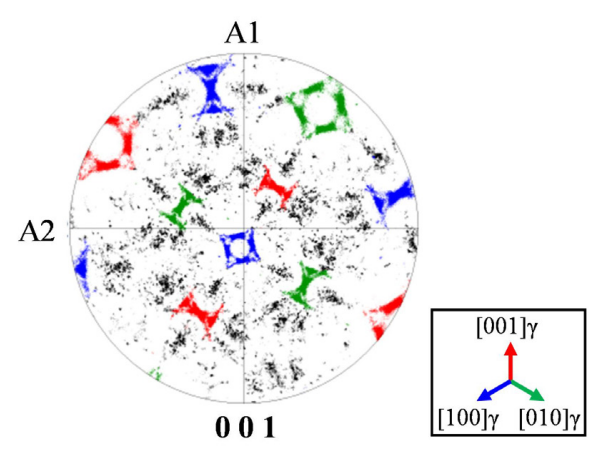

(b) Pole Figure of $<001>a$

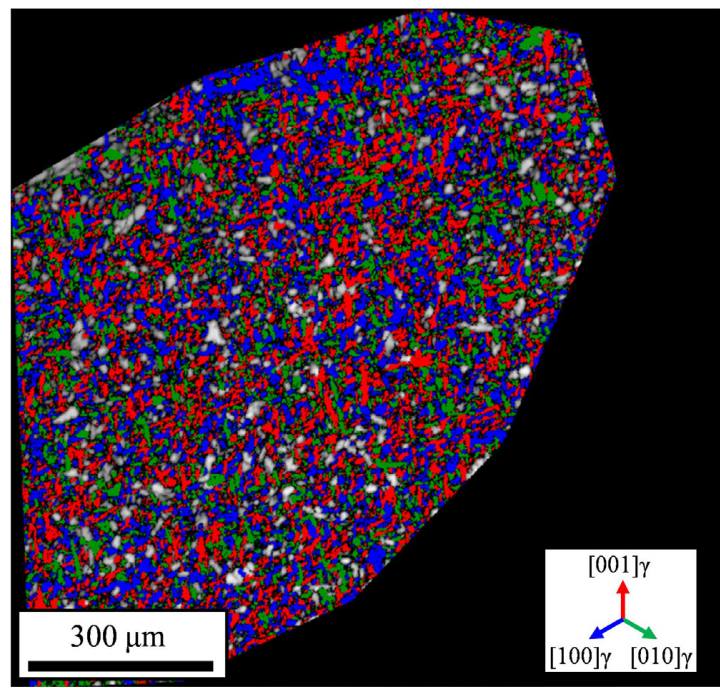

(c) Bain Map

Fig. 8 Crystal Orientation Analysis by EBSD 
ferrite precipitated from the inside. However, it is possible that before prior $\gamma$ grains completely transformed into intragranular idiomorphic ferrite, the system was cooled to a temperature below which acicular ferrite precipitates to finally form a mixed structure of intragranular idiomorphic and acicular ferrites.

\section{Conclusions}

In the present study, we observed metallographic structures in the weld metal of a joint welded by high-current buried-arc welding and found three regions with different metallographic structures. We focused on the most characteristic textures near the fusion boundary for detailed observations and discussions. The results are summarized as follows:

(1) Although the macrostructure appears extremely coarse, it is considered to be a trace of the prior $\gamma$ grain, whereas the microstructure is mainly fine intragranular ferrite texture.

(2) Detailed analysis of the intragranular ferrite via crystal orientation analysis using EBSD showed that it was a mixed structure of ferrites that did not satisfy the K-S OR with the prior $\gamma$ grain and ferrites that satisfied the K-S OR. The prior is considered to be intragranular idiomorphic ferrite that precipitates at a relatively high temperature, whereas the latter is acicular ferrite that precipitates at a relatively low temperature.

(3) Extremely coarse prior $\gamma$ grains and a mixed microstructure of acicular ferrite and intragranular idiomorphic ferrite were presumably caused by the high heat input of the high-current buried-arc welding.

\section{References}

1) H. Baba, T. Era, K. Maeda and H. Terasaki: Basic study on welding of low carbon steel using high current buried arc - Development of GMA welding system with ultra-high wire feed rate (Third report)-, Preprints of the National Meeting of JWS, 102 (2018), 178-179. (in Japanese)

2) H. Baba, T. Era, T. Ueyama and M. Tanaka: Single pass full penetration joining for heavy plate steel using high current GMA process, Welding in the World, 61-5 (2017), 963-969.

3) S. S. Babu and S. A. David: Inclusion Formation and Microstructure Evolution in Low Alloy Steel Welds, ISIJ International, 42-12 (2002), 1344-1353.

4) K. Koumatos and A. Muehlemann: A Theoretical Investigation of Orientation Relationships and Transformation Strains in Steels, Acta Cryst. , A73 (2017), 115-123.

5) K. Kwak, T. Mayama, Y. Mine and K. Takashima: Micro-tensile Behaviour of Low-alloy Steel with Bainite/martensite Microstructure, ISIJ International, 56-12 (2016), 2313-2319.

6) H. Terasaki, Y. Shintome, Y. Komizo, M. Ohata, K. Moriguchi and Y. Tomio: Effect of Close-Packed Plane Boundaries in a Bain Zone on the Crack Path in Simulated Coarse-Grained HAZ of Bainitic Steel, Metallurgical and Materials Transactions A, 46-5 (2015), 2035-2039.

7) G. Miyamoto, T. Shinyoshi, J. Yamaguchi, T. Furuhara, T. Maki and R. Uemori: Crystallography of intragranular ferrite formed on $(\mathrm{MnS}+\mathrm{V}(\mathrm{C}, \mathrm{N}))$ complex precipitate in austenite, Scripta Materialia, 48 (2003), 371-377.

8) M. Wahba, M. Mizutani and S. Katayama: Microstructure and Mechanical Properties of Hybrid Welded Joints with Laser and $\mathrm{CO}_{2}$-Shielded Arc, Journal of Materials Engineering and Performance, 25-7 (2016), 2889-2894. 